\title{
Inhibition of mitochondrial remodeling by cyclosporine A preserves myocardial performance in a neonatal rabbit model of cardioplegic arrest
}

\author{
Norihiko Oka, MD, Lixing Wang, MD, PhD, Wenyu Mi, MD, and Christopher A. Caldarone, MD
}

The Division of Cardiovascular Surgery at the Hospital for Sick Children, University of Toronto, Toronto, Ontario, Canada.

Received for publication Aug 21, 2007; revisions received Sept 12, 2007; accepted for publication Sept 24, 2007.

Address for reprints: Christopher A. Caldarone, MD, Division of Cardiovascular Surgery, The Hospital for Sick Children, 555 University Ave, Toronto, Ontario, Canada M5G 1X8 (E-mail: christopher.caldarone@ sickkids.ca).

J Thorac Cardiovasc Surg 2008;135:585-93 $0022-5223 / \$ 34.00$

Copyright $\odot 2008$ by The American Association for Thoracic Surgery

doi:10.1016/j.jtcvs.2007.09.023
Objective: Mitochondrial permeability transition pore opening is associated with apoptotic signaling and alterations in mitochondrial structure and function. We tested whether inhibition of mitochondrial permeability transition pore opening with cyclosporine A preserved mitochondrial structure and function after cardioplegic arrest and whether this preservation is associated with improved myocardial performance.

Methods: Langendorff-perfused neonatal rabbit hearts were perfused for 30 minutes with Krebs-Henseleit buffer (CCP; $n=6)$ or Krebs-Henseleit buffer containing 2 $\mu \mathrm{mol} / \mathrm{L}$ of cyclosporine $\mathrm{A}(\mathrm{CCP}+\mathrm{CsA} ; \mathrm{n}=6)$ followed by 60 minutes of normothermic crystalloid cardioplegia (CCP) and 60 minutes of reperfusion. Control hearts (non$\mathrm{CCP} ; \mathrm{n}=6$ ) were constantly perfused for 150 minutes without cardioplegic arrest.

Results: In comparison with non-CCP, CCP was associated with Bax translocation to the mitochondria, cytochrome c release, and greater frequency of terminal deoxynucleotidyl transferase-mediated dUTP nick end labeling-positive myocytes These changes were also associated with deficits in isolated mitochondrial oxygen consumption at complex I. CsA pretreatment minimized or prevented all these findings. Myocardial performance (systolic pressure, maximum positive and negative $\mathrm{dP} / \mathrm{dt}$, and elevated left ventricular end-diastolic pressure) at 5, 15, 30, and 60 minutes after reperfusion was diminished in $\mathrm{CCP}$ hearts when compared with non-CPB, and these deficits could be minimized with cyclosporine A pretreatment. $(P<.05$ all comparisons)

Conclusions: Cyclosporine A prevents apoptosis-related mitochondrial permeabilization and dysfunction after cardioplegic arrest. This protection is associated with improved myocardial performance. Prevention of mitochondrial permeability transition pore opening is a valuable target for mitochondrial (and myocardial) preservation after neonatal cardioplegic arrest.

A $\mathrm{s}$ a critical mediator in apoptosis signaling pathways, mitochondria integrate upstream death stimuli and undergo structural and functional remodeling with subsequent transmission of signals to downstream executioner proteins. ${ }^{1}$ Bax translocation, mitochondrial outer membrane permeabilization, and cytochrome c release directly participate in mitochondrial architectural and functional remodeling after unprotected ischemia/reperfusion. ${ }^{2-4}$ After protected ischemia/reperfusion (cardioplegic arrest), we have previously reported a similar constellation of apoptosis-related alterations in mitochondrial structure and function in the lamb and piglet myocardium (apoptosis-related mitochondrial dysfunction: ARMD). ${ }^{5-7}$ We correlated these changes with deficits in mitochondrial electronic transport chain activity. ${ }^{89}$ Because of the close interplay between apoptotic events and mitochondrial dysfunction, as well as the role of mitochondrial protection in the maintenance of cardiac performance, ${ }^{10}$ we hypothesized that "mitoprotection" conferred by maintenance of outer mitochondrial membrane integrity may be of therapeutic benefit to reduce ARMD and consequently preserve myocardial performance.

The mitochondrial permeability transition pore (MPTP) is a nonspecific pore that may mediate alterations in outer mitochondrial membrane integrity. ${ }^{11,12}$ 


\author{
Abbreviations and Acronyms \\ ARMD = apoptosis-related \\ mitochondrial dysfunction \\ BSA $=$ bovine serum albumin \\ $\mathrm{CCP}=$ crystalloid cardioplegia \\ COX IV = cytochrome $\mathrm{c}$ oxidase IV \\ CsA $=$ cyclosporine $\mathrm{A}$ \\ $\pm \mathrm{dP} / \mathrm{dtmax}=$ maximum first derivative \\ of left ventricular pressure \\ IgG = immunoglobulin $\mathrm{G}$ \\ $\mathrm{KH} \quad=$ Krebs-Henseleit buffer \\ $\mathrm{LV} \quad=$ left ventricular \\ MIB = mitochondrial isolation buffer \\ MPTP $=$ mitochondrial permeability \\ transition pore \\ TMPD $=N, N, N^{\prime} N^{\prime}$-tetramethyl- \\ p-phenylenediamine \\ TUNEL = terminal deoxynucleotidyl \\ transferase-mediated \\ dUTP nick end labeling
}

Pharmacologic inhibition of MPTP opening is associated with myocardial protection against unprotected ischemia/reperfusion injury. ${ }^{13,14}$ Cyclosporine A (CsA), a specific inhibitor of MPTP opening, acts by inhibiting the peptidyl-prolyl cis-trans isomerase activity of the cyclophilin-D component of the MPTP and has been reported to ameliorate reperfusion injury in a variety of in vitro ${ }^{15,16}$ and in vivo ${ }^{17}$ animal models of unprotected and protected ischemia. ${ }^{7,18}$

$\mathrm{We}^{7}$ have previously reported that CsA-mediated MPTP pore inhibition prevents ARMD in a neonatal piglet model. This report extends these observations in a neonatal rabbit model and relates amelioration of ARMD to preservation of global myocardial performance.

\section{Methods and Materials}

\section{Experimental Model}

New Zealand White neonatal rabbits (5-6 days; 150-250 g) were anesthetized with sodium pentobarbital $(50 \mathrm{mg} / \mathrm{kg}$ intraperitoneally), heparinized (1000 U/kg intraperitoneally), and ventilated. The heart was retrogradely perfused in situ to avoid ischemia, excised, mounted on a Langendorff perfusion system, and perfused at $75 \mathrm{~mm} \mathrm{Hg}$ with Krebs-Henseleit buffer $(\mathrm{KH})$ equilibrated with $95 \%$ oxygen and $5 \%$ carbon dioxide ( $\mathrm{pH}$ of 7.35-7.40).

All animals received humane care in compliance with the "Guide for the Care and Use of Laboratory Animals" prepared by the Association for Assessment and Accreditation of Laboratory Animal Care (AAALAC; March 1999).

\section{Experimental Protocol}

Two groups of hearts were perfused for a 30-minute stabilization period followed by 60 minutes of crystalloid cardioplegic arrest (CCP) and 60 minutes of reperfusion. During the stabilization period, cyclosporine A (CsA)-treated hearts $(\mathrm{CCP}+\mathrm{CsA}$ group, $\mathrm{n}=6)$ were perfused with $\mathrm{KH}$ in which $\mathrm{CsA}(0.2 \mu \mathrm{mol} / \mathrm{L})$ was added to the KH. Nontreated hearts (CCP, $n=6)$ were stabilized with $\mathrm{KH}$ perfusion alone. Cardioplegic arrest for both groups was induced with crystalloid cardioplegia $(65 \mathrm{~mL} / \mathrm{kg}$, Plegisol; Hospira, Inc, Lake Forest, Ill) at normothermia. ${ }^{19} \mathrm{CsA}$ was dissolved in dimethylsulfoxide (Fisher Scientific, Waltham, Mass), which was further dissolved into $\mathrm{KH}$ at a final concentration of $0.05 \%$. The same amount of dimethylsulfoxide was added into the $\mathrm{KH}$ for all other groups.

A third group of hearts underwent uninterrupted perfusion (non$\mathrm{CCP}, \mathrm{n}=6$ ) for 150 minutes.

At the end of reperfusion, the hearts were rapidly excised. For mitochondrial oxygen consumption and mitochondrial protein extraction, myocardium was fractionated as described. ${ }^{8}$

A separate set of animals ( $n=4$ for each group) underwent identical procedures after which the left ventricles were sliced and embedded in OCT compound (IMEB, Inc, San Marcos, Calif), snap frozen in liquid nitrogen, and stored at $-80^{\circ} \mathrm{C}$ for apoptosis and fluorescence immunohistochemistry.

\section{Cardiac Functional Measurements}

Isovolumetric measurement of left ventricular (LV) performance was made with a water-filled balloon connected to a pressure transducer (MLT844; ADInstruments, Colorado Springs, Colo) and inserted into the left ventricle across the mitral valve. The volume of the water-filled balloon was adjusted to an end-diastolic pressure of 0 to $5 \mathrm{~mm} \mathrm{Hg}$ and kept constant throughout the entire experiment. LV performance was assessed with LV systolic pressure, LV enddiastolic pressure, LV developed pressure, and maximum positive and negative derivative of LV pressure ( $\pm \mathrm{dP} / \mathrm{dtmax})$. Analog data (heart rate and LV systolic pressure, developed pressure, enddiastolic pressure, and $\pm \mathrm{dP} / \mathrm{dtmax}$ ) were digitalized and analyzed (Chart IV; ADInstruments, Colorado Springs, Colo). Hemodynamic data were compared at comparable time points in all three groups.

\section{Mitochondrial Isolation}

The left ventricle was used for mitochondrial isolation by differential centrifugation. ${ }^{20}$ The whole left ventricle $(\sim 0.7 \mathrm{~g})$ was placed into 5 $\mathrm{mL}$ of mitochondrial isolation buffer (MIB) on ice $(5 \mathrm{mmol} / \mathrm{L}$ MOPS, $2 \mathrm{mmol} / \mathrm{L}$ ethylene glycol bis-2-aminoethyl etherN,N',N",n'-tetraacetic acid, $70 \mathrm{mmol} / \mathrm{L}$ sucrose, $220 \mathrm{mmol} / \mathrm{L}$ mannitol, $1 \mathrm{mmol} / \mathrm{L}$ dithiothreitol, $17 \mu \mathrm{g} / \mathrm{mL}$ phenylmethylsulfonyl fluoride, $8 \mu \mathrm{g} / \mathrm{mL}$ aproptinin, and $2 \mu \mathrm{g} / \mathrm{mL}$ leupeptin, $\mathrm{pH} 7.2$ ) with $0.1 \%$ bovine serum albumin (BSA) and finely minced. Tissue was homogenized on ice by a blade homogenizer and centrifuged (700 $g \times 5$ minutes). The supernatant was transferred to a new tube and the same low spin repeated four times ( 5 minutes each) until no visible pellet was seen. The supernatant from the low spins was centrifuged $(8000 \mathrm{~g} \times 10$ minutes). The pellet was resuspended in $10 \mathrm{~mL}$ MIB with $0.1 \%$ BSA and centrifuged for another 10 minutes $(8000 \mathrm{~g})$. The final pellet was suspended in MIB buffer without BSA for mitochondrial oxygen consumption measurements.

\section{Clark-electrode Oxygen Consumption Measurement}

Mitochondrial complex I, II, and IV respiration was measured with a Clark-type oxygen electrode (Instech Laboratories Inc, Plymouth Meeting, Pa). Oxygen consumption was measured in the presence of sequential administration of substrates and inhibitors (glutamate/ malate for complex I, rotenone/succinate for complex II, 

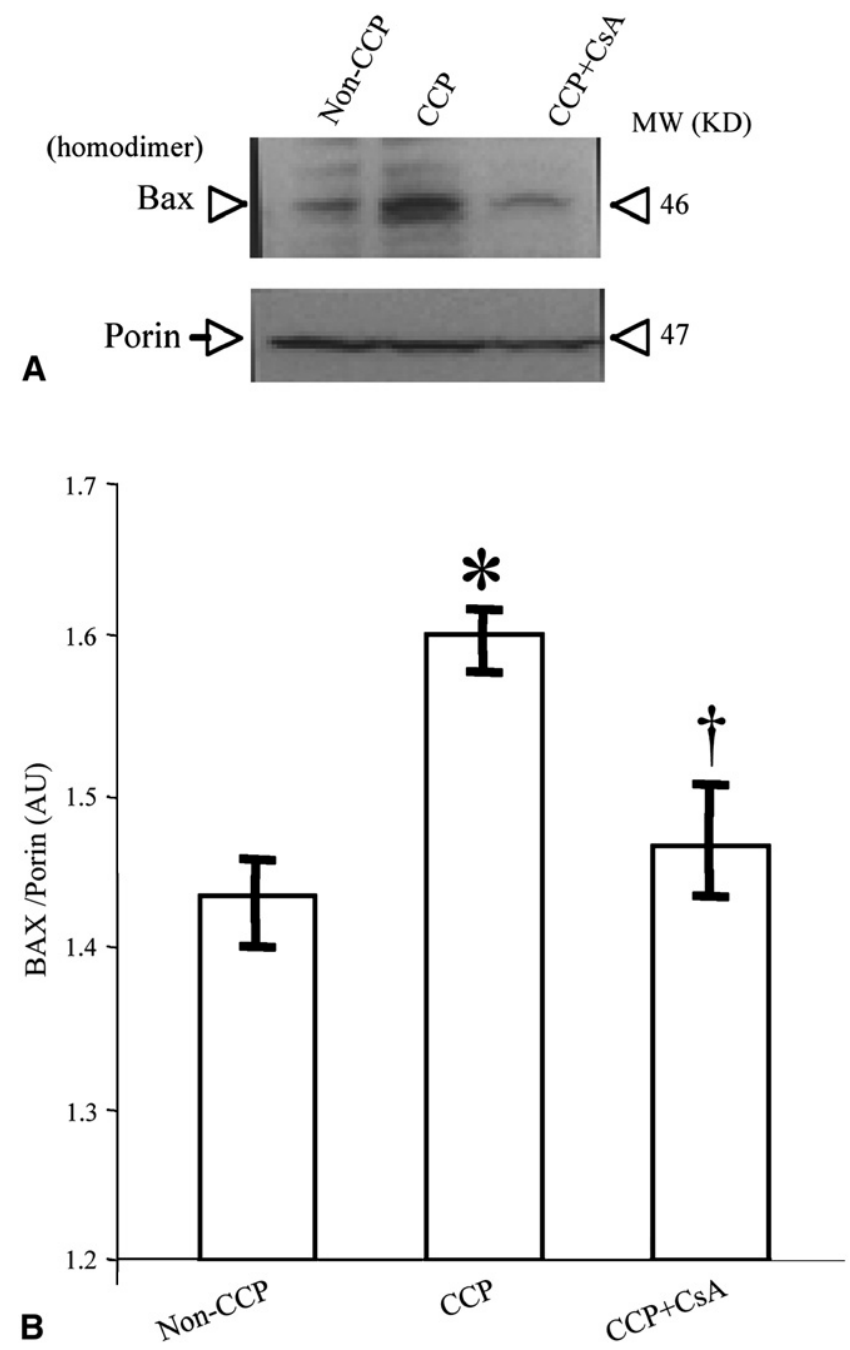

Figure 1. A, Representative Western blot of mitochondrial Bax in non-CCP, CCP, and CCP+CsA myocardium. B, Densitometric measurements of Bax in non-CCP, CCP, and CCP+CsA hearts. Increased concentrations of Bax (homodimer) in the mitochondrial fractions normalized by porin content are indicative of translocation of Bax to the mitochondria. ${ }^{*} \boldsymbol{P}<.01$ versus non-CCP; $\dagger P<.05$ versus CCP. CCP, Crystalloid cardioplegia; $C s A$, cyclosporine $A$; $M W$, molecular weight; $A U$, arbitrary units.

antimycin/TMPD/ascorbate for complex IV) added in the following order and final concentration: $2.5 \mathrm{mmol} / \mathrm{L}$ glutamate, $2.5 \mathrm{mmol} / \mathrm{L}$ malate, $2 \mathrm{mmol} / \mathrm{L}$ adenosine diphosphate, $2 \mu \mathrm{mol} / \mathrm{L}$ rotenone, 5 $\mathrm{mmol} / \mathrm{L}$ succinate, $1 \mu \mathrm{mol} / \mathrm{L}$ antimycin $\mathrm{A}$, and $1 \mathrm{mmol} / \mathrm{L}$ ascorbate with $\quad 0.4 \mathrm{mmol} / \mathrm{L} \quad N, N, N^{\prime} N^{\prime}$-tetramethyl-p-phenylenediamine (TMPD). Respiration rates are expressed as micromoles of oxygen per minute per milligram of mitochondrial protein.

\section{Mitochondrial Bax Translocation}

Mitochondrial pellets were lysed in a hypotonic buffer $(250 \mathrm{mmol} / \mathrm{L}$ sucrose, $20 \mathrm{mmol} / \mathrm{L} \mathrm{HEPES,} 10 \mathrm{mmol} / \mathrm{L} \mathrm{KCl}, 1.5 \mathrm{MgCl}_{2}, 1 \mathrm{mmol} / \mathrm{L}$ ethylenediaminetetraacetic acid, $1 \mathrm{mmol} / \mathrm{L}$ ethyleneglycol-bis-
( $\beta$-aminoethylether)- $N, N, N^{\prime}$ tetraacetic acid, $1 \mathrm{mmol} /$ Lphenylmethylsulfonyl fluoride, $8 \mu \mathrm{g} / \mathrm{mL}$ aproptinin, and $2 \mu \mathrm{g} / \mathrm{mL}$ leupeptin [pH 7.4]). After incubation for 30 minutes, the suspension was sonicated and recentrifuged $(13,000 \mathrm{~g} \times 30$ minutes $)$. The soluble proteins were used for measurement of Bax translocation by Western blot. Membranes were probed with anti-rabbit Bax (N-20, 1:1000; Santa Cruz Biotechnology, Inc, Santa Cruz, Calif). For protein loading control, the membranes were stripped and reprobed with antimouse mitochondrial porin (1:20,000; Molecular Probes, Inc, Eugene, Ore). Secondary antibodies were coupled to horseradish peroxidase (anti-rabbit immunoglobulin G [IgG] 1:1000; anti-mouse IgG 1:10,000; Santa Cruz Biotechnology).

\section{Fluorescent Immunohistochemistry and Confocal Imaging}

OCT-embedded transverse ventricular slices were cut into $5-\mu \mathrm{m}$ serial sections, fixed in acetone, and blocked. Mouse monoclonal anti-cytochrome c oxidase IV (COX IV) (1:250; BD Biosciences Clontech, Palo Alto, Calif) was used. Sections were incubated with secondary antibody (Cy3-conjugated donkey anti-mouse IgG, 1:500; Jackson ImmunoResearch Laboratories, Inc, West Grove, Pa). Mouse monoclonal anti-cytochrome c (1:200; BD Biosciences Pharmingen, San Diego, Calif) was used as the primary antibody for cytochrome c staining. Sections were immersed in secondary antibody (Cy2-conjugated donkey anti-mouse IgG, 1:200; Jackson ImmunoResearch Laboratories).

Images from at least three different sections were acquired at $64 \times$ by a Zeiss LSM510 Multiphoton Laser Scanning Confocal Microscope (Carl Zeiss, Jena, Germany) with the same pinhole setting, pixel format $(1024 \times 1024)$, and scanning data depth $(0.8$ $\mu \mathrm{m})$. Double fluorescence for green and red channels was imaged by excitation of argon-HeNe1 at wavelengths of 488 and $530 \mathrm{~nm}$. Fifty high-power fields from each animal were analyzed by imaging processing software (Volocity 3.0; Improvision, Lexington, Mass). High intensity of red and green (above 551 voxels) was regarded as specific immunoreactive signals. The red (COX IV) was set as reference signal and the contribution of the green (cytochrome c) to the colocalization of the both signals was quantified as the overlap coefficient (Kx-green) and used to compare the diffusion of cytochrome $\mathrm{c}$ staining between groups. ${ }^{21}$

\section{Terminal Deoxynucleotidyl Transferase-mediated dUTP Nick End Labeling (TUNEL) Staining}

OCT-embedded transverse ventricular slices were cut into $5-\mu \mathrm{m}$ serial sections. TUNEL staining was performed with the In Situ Cell Death Detection Kit (Roche Diagnostics, Indianapolis, Ind) according to the manufacturer's instructions. After TUNEL, all nuclei were counterstained with 4,6-diamino-2-phenylindole. TUNELpositive myocytes and the number of total myocytes were counted in 50 random high-power fields $(\times 600)$ with a Leica (Deerfield, Ill) fluorescent microscope and OpenLab software (Improvision, Lexington, Mass). Approximately 10,000 myocytes were examined from at least three different slices from each animal.

\section{Statistics}

Data are expressed as mean \pm SEM and group comparisons made with the Fisher least significant difference analysis of variance. A Tukey test was used for multiple post hoc comparisons. 

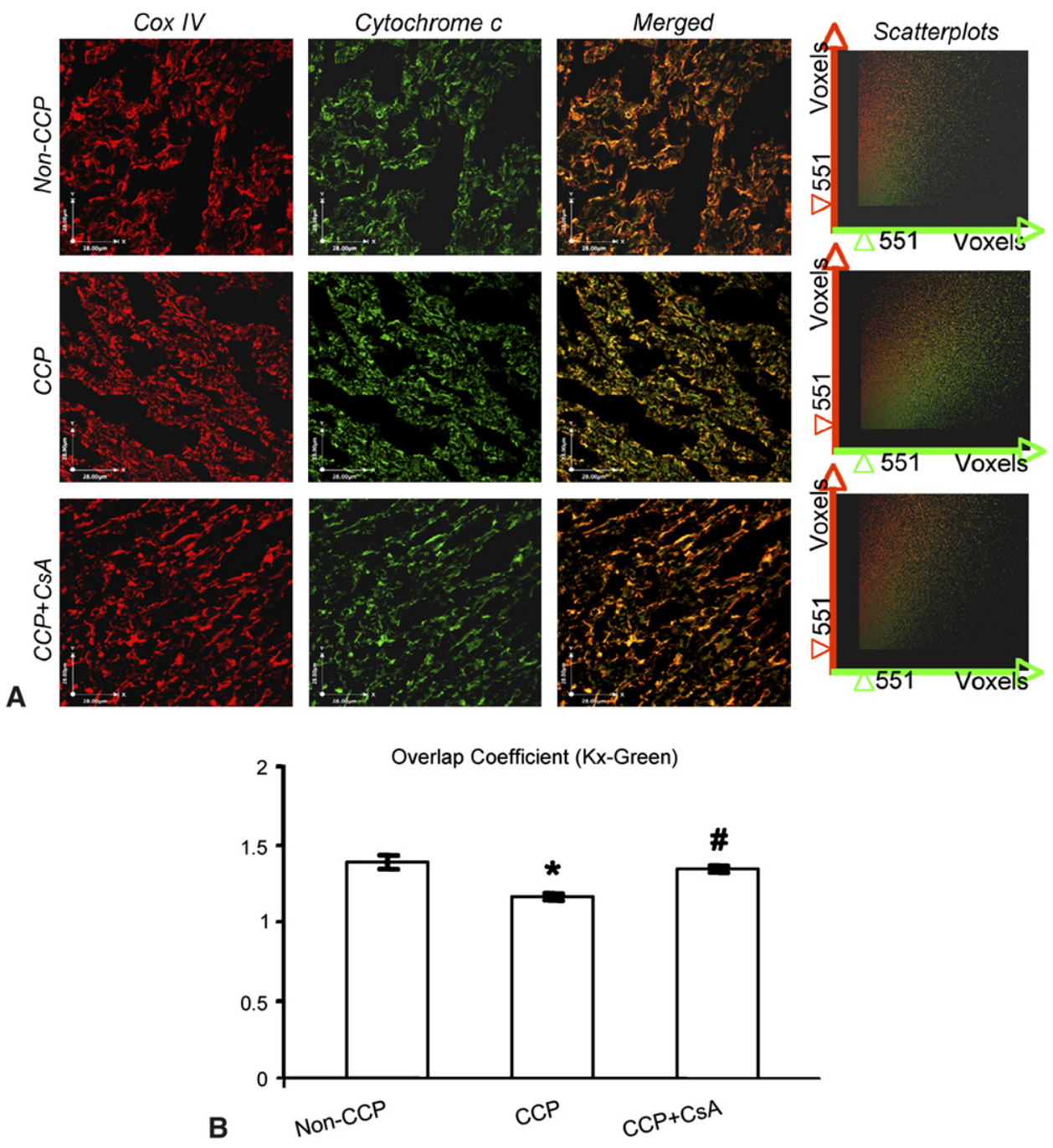

Figure 2. A, Immunofluorescence of cytochrome c oxidase IV (COX IV) and cytochrome $c$ in the myocardium from non-CCP, CCP, and CCP+CsA myocardium. In each panel, COX IV (mitochondria) is stained red, cytochrome $c$ is stained green, and the merged mages are shown. Superimposition of red and green staining results in a brownish color that suggests retention of cytochrome $c$ in the non-CCP and CCP + CsA mitochondria. A fine diffuse green staining can be seen in the merged images of CCP myocardium, suggesting mitochondrial release of cytochrome c. Scatterplots display the distribution of red and green voxels in the merge images of non-CCP, CCP, and CCP+CsA, as well as the various degrees of colocalization as shown in orange and yellow. B, Quantification of the overlap coefficient (Kx-green) in the merge images for non-CCP, CCP, and CCP+CsA groups is shown. ${ }^{*} P<.01$ versus non-CCP; \#P<.01 versus CCP. For abbreviations, see Figure 1.

\section{Results}

\section{Mitochondrial Bax Translocation}

CCP was associated with Bax translocation to the mitochondria (homodimer) in the mitochondrial protein fraction when compared with the non-CCP myocardium $(1.60 \pm 0.02$ vs $1.43 \pm 0.30$ arbitrary units, $P<.01)$. Bax translocation was reduced after CsA treatment (1.47 \pm 0.04 arbitrary units; $P<.05$ vs CCP) to the levels that were similar to non-CCP (Figure 1).

\section{Mitochondrial Permeabilization and Cytochrome c} Release

Fluorescence immunohistochemistry was used to compare patterns of cytochrome $\mathrm{c}$ and COX IV staining (Figure 2). Release of cytochrome $\mathrm{c}$ from the mitochondria causes diffusion of the cytochrome c staining in the cytoplasm and a stippled green pattern is produced. This pattern is prominent in the merged images from the CCP myocardium, whereas in non-CCP and CCP+CsA myocardium, only faint diffusion 

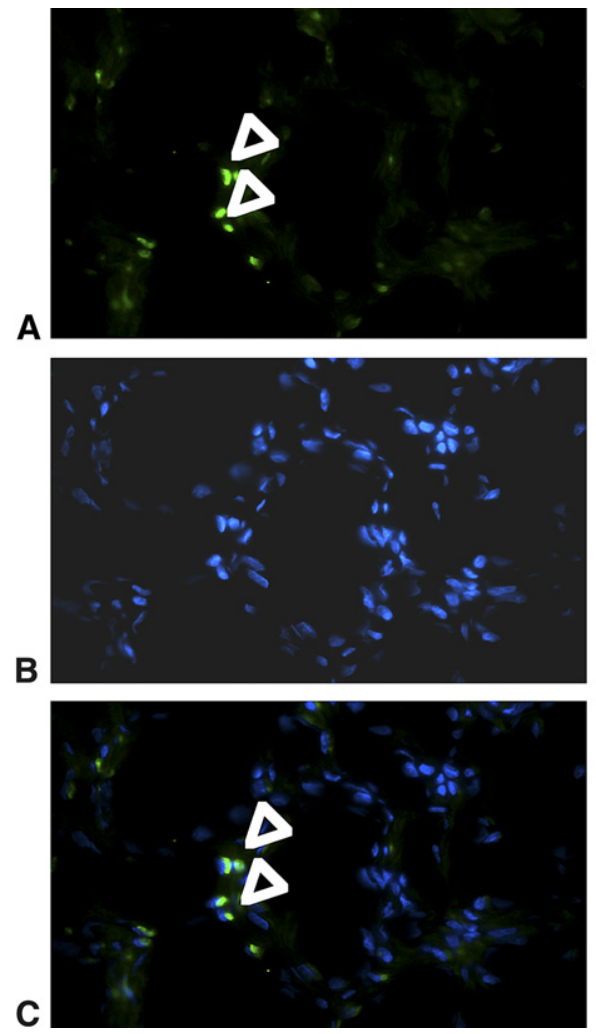

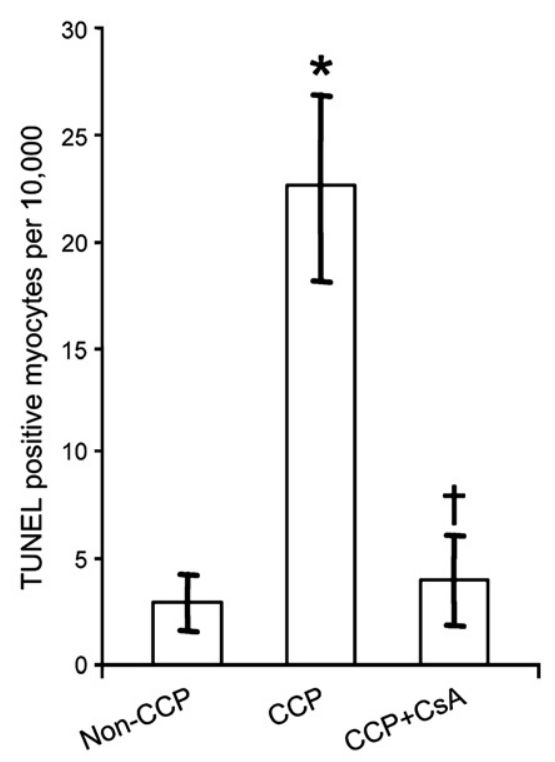

Figure 3. Representative imaging of TUNEL staining. A, TUNEL-positive nuclei (green), which are indicated by white arrows. B, Nuclei 4,6-diamino-2-phenylindole staining (blue). C, A merged picture of $A$ and $B$. $D$, Quantitative analysis of TUNEL staining for non-CCP, CCP, and CCP+CsA myocardium. ${ }^{*} P<.01$ versus non-CPB; $\dagger P<.05$ versus CCP. For abbreviations, see Figure 1. of cytochrome c staining in the merged images was found. The contribution of the green color intensity to the colocalization of these color voxels in the merged images was quantified by overlap coefficients (Kx-green). Compared with non-CCP and $\mathrm{CCP}+\mathrm{Cs}$ A myocardium, lower overlap coefficient $(\mathrm{Kx}$-green) was found in CCP myocardium $(P<.01)$.

\section{TUNEL Staining}

TUNEL-positive nuclei were more frequent in CCP myocardium than in non-CCP and $\mathrm{CCP}+\mathrm{CsA}$ myocardium $(22.5 \pm$ 4.5 vs $3.0 \pm 1.3$ and $4.0 \pm 2.2$ per 10,000 nuclei; $P<.01$ ) (Figure 3). There was no difference between non-CCP and $\mathrm{CCP}+\mathrm{CsA}$ groups $(P=.82)$.

\section{Clark-electrode Oxygen Consumption Measurements}

Isolated mitochondrial oxygen consumption was assessed during state 2 respiration for mitochondrial complexes I, II, and IV and was not different among groups, suggesting comparable levels of mitochondrial integrity after fractionation. Cardioplegic arrest was associated with diminished state 3 respiration, and the decrement of state 3 and the ratio of state 3 to state 2 was prevented by pretreatment with CsA $(P<.01)$ (Figure 4 and Table 1). Complex II and complex IV oxygen consumption rates were not different between groups.

\section{Myocardial Performance}

There was no significant different change in heart rate among non-CCP, CCP, and CCP+CsA. LV developed systolic pressure and $\pm \mathrm{dP} / \mathrm{dtmax}$ were significantly depressed at 5 , 15,30 , and 60 minutes of reperfusion in the CCP hearts (compared with non-CCP). LV end-diastolic pressure was significantly increased in CCP when compared with nonCCP. In contrast, administration of CsA improved all these parameters (Table 2 and Figure 5).

\section{Discussion}

A typical pathologic change in the postischemic myocardium after cardioplegic arrest is manifest by a constellation of apoptotic events ${ }^{19}$ and apoptosis-related alterations in mitochondrial structure and function including Bax translocation, mitochondrial permeabilization, cytochrome $\mathrm{c}$ release, and deficits in mitochondrial electron transport. ${ }^{8,9}$ We have previously defined this constellation of events as ARMD. The current study demonstrates that pretreatment with CsA, an MPTP inhibitor, prevents ARMD and this prevention is associated with preservation of global myocardial performance.

The etiology of mitochondrial permeabilization during apoptosis is not fully determined. Bax translocation, however, is associated with disruption of outer mitochondrial 

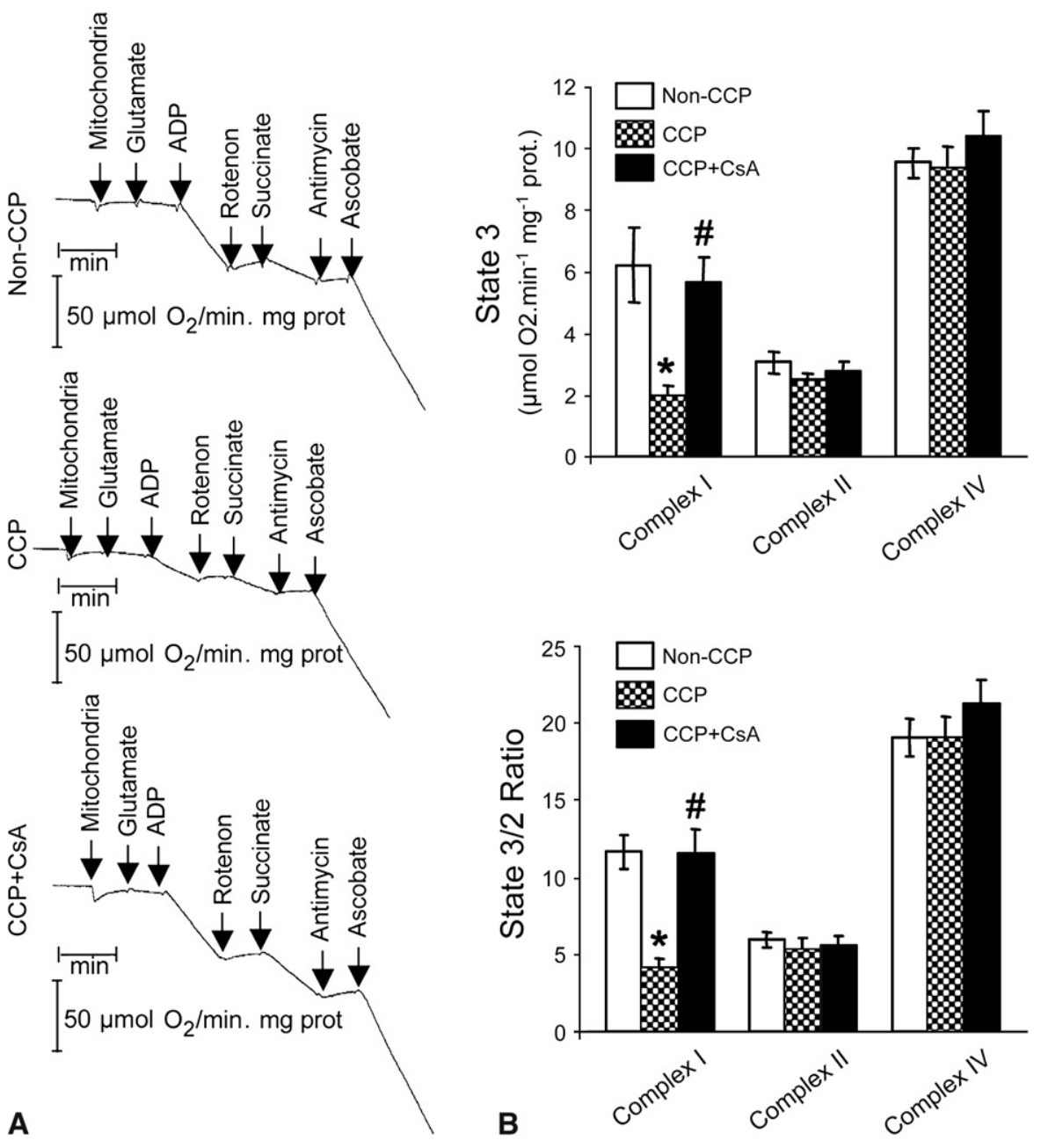

Figure 4. $A$, The representative traces of real-time mitochondrial oxygen consumption including complexes I, II, and IV from non-CCP, CCP, and CCP+CsA myocardium. B, Comparison of the activity of state 3 and the ratio of state 3 to state 2 of complexes I, II, and IV among the groups. ${ }^{*} P<.01$ versus non-CCP; \#P $<.01$ versus CCP. $A D P$, Adenosine diphosphate. For other abbreviations, see Figure 1.

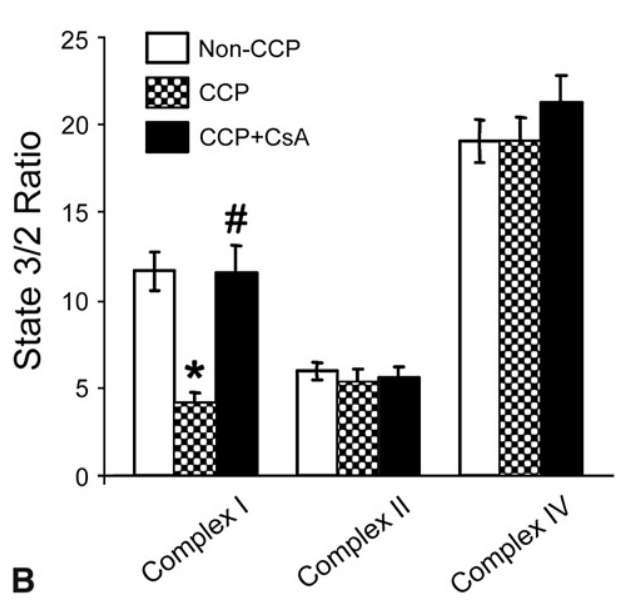

membrane integrity and cytochrome $\mathrm{c}$ release into the cytoplasm. The mechanism by which Bax induces the permeabilization is not clearly defined, and it may act through direct pore-forming capacity ${ }^{22}$ or through direct interaction with the MPTP. ${ }^{23}$ Alternatively, transient MPTP opening may act as an initial event to stimulate Bax translocation to the mitochondria ${ }^{24}$ and, through a positive feedback loop between Bax and the MPTP, stimulate further permeabilization and cytochrome c release. ${ }^{25}$ In the present study,
CsA prevented Bax translocation, suggesting that MPTP opening is an "upstream" signaling event that is required for Bax translocation and subsequent mitochondrial permeabilization.

Cytochrome $\mathrm{c}$ is an important component of mitochondrial oxidative phosphorylation. A relative deficiency of mitochondrial cytochrome $\mathrm{c}$, as may occur after mitochondrial permeabilization, may be responsible for deficits in mitochondrial respiration. ${ }^{2}$ The immunohistochemistry data

TABLE 1. Isolated mitochondrial oxygen consumption ( $\mu \mathrm{mol}$ oxygen $\cdot \mathrm{min}^{-1} \cdot \mathrm{mg}^{-1}$ protein) of non-CCP, CCP, and CCP $+\mathrm{CsA}$ mitochondria

\begin{tabular}{|c|c|c|c|c|c|c|c|c|c|}
\hline & \multicolumn{3}{|c|}{ Complex I } & \multicolumn{3}{|c|}{ Complex II } & \multicolumn{3}{|c|}{ Complex IV } \\
\hline & Non-CCP & CCP & $\mathrm{CCP}+\mathrm{CsA}$ & Non-CCP & CCP & $\mathrm{CCP}+\mathrm{CsA}$ & Non-CCP & CCP & $\mathrm{CCP}+\mathrm{CsA}$ \\
\hline & $0.52 \pm$ & $0.51 \pm 0.05$ & $0.49 \pm 0.02$ & $0.52 \pm 0.05$ & $0.51 \pm 0.05$ & $0.49 \pm 0.02$ & $0.52 \pm 0.05$ & $0.51 \pm 0.05$ & $0.49 \pm$ \\
\hline State 3 & $6.21 \pm 1.21$ & $2.00 \pm 0.28^{*}$ & $5.64 \pm 0.82 \dagger$ & $3.05 \pm 0.38$ & $2.52 \pm 0.19$ & $2.77 \pm 0.30$ & $9.53 \pm 0.47$ & $9.39 \pm 0.66$ & $10.42 \pm 0.81$ \\
\hline Ratio 3/2 & $11.65 \pm 1.15$ & $4.08 \pm 0.68^{*}$ & $11.48 \pm 1.62 \dagger$ & $5.93 \pm 0.45$ & $5.29 \pm 0.76$ & $5.62 \pm 0.54$ & $19.06 \pm 1.24$ & $19.07 \pm 1.32$ & $21.24 \pm 1.62$ \\
\hline
\end{tabular}

CCP, Cardioplegic arrest; $C s A$, cyclosporine A. ${ }^{*} P<.01$ versus non-CCP; $\dagger P<.01$ versus CCP. 
TABLE 2. Hemodynamics in non-CCP, CCP, and CCP+CsA treated hearts

\begin{tabular}{|c|c|c|c|c|c|c|c|c|c|c|c|c|}
\hline \multirow[b]{3}{*}{ Variables } & \multirow{2}{*}{\multicolumn{3}{|c|}{ Baseline }} & \multicolumn{9}{|c|}{ Reperfused } \\
\hline & & & & \multicolumn{3}{|c|}{15 minutes } & \multicolumn{3}{|c|}{30 minutes } & \multicolumn{3}{|c|}{60 minutes } \\
\hline & Non-CCP & CCP & $\mathrm{CCP}+\mathrm{CsA}$ & Non-CCP & CCP & $\mathrm{CCP}+\mathrm{CsA}$ & Non-CCP & CCP & $\mathrm{CCP}+\mathrm{CsA}$ & Non-CCP & CCP & $\mathrm{CCP}+\mathrm{CsA}$ \\
\hline HR (beats/min) & $210 \pm 6$ & $230 \pm 7$ & $213 \pm 6$ & $190 \pm 12$ & $230 \pm 8$ & $203 \pm 8$ & $180 \pm 11$ & $236 \pm 9$ & $203 \pm 8$ & $183 \pm 1$ & $230 \pm 6$ & $196 \pm 8$ \\
\hline $\operatorname{LVDP}(\Delta \%)$ & 100 & 100 & 100 & $97 \pm 1$ & $80 \pm 1^{*}$ & $90 \pm 3 \dagger$ & $95 \pm 1$ & $84 \pm 0.7^{*}$ & $95 \pm 1.2 \dagger$ & $95 \pm 1$ & $77 \pm 1^{*}$ & $94 \pm 2 \dagger$ \\
\hline LVEDP $(\mathrm{mm} \mathrm{Hg})$ & $3.0 \pm 0.2$ & $3.1 \pm 0.0$ & $2.9 \pm 0.1$ & $3.1 \pm 0.3$ & $17 \pm 1.8^{*}$ & $6.7 \pm 0.9 \dagger$ & $3.0 \pm 0.2$ & $11.7 \pm 1.6^{*}$ & $3.6 \pm 0.2 \dagger$ & $3.2 \pm 0.3$ & $11.4 \pm 1.1^{*}$ & $3.2 \pm 0.3 \dagger$ \\
\hline$+\mathrm{dP} / \mathrm{dtmax}(\Delta \%)$ & 100 & 100 & 100 & $95 \pm 0.9$ & $73 \pm 2.6^{*}$ & $86 \pm 3.7^{*} \dagger$ & $95 \pm 1.1$ & $80 \pm 3.3^{*}$ & $94 \pm 1.8 \dagger$ & $94 \pm 1.6$ & $74 \pm 3.4^{*}$ & $92 \pm 2.3 \dagger$ \\
\hline$-\mathrm{dP} / \mathrm{dtmax}(\Delta \%)$ & 100 & 100 & 100 & $95 \pm 1.2$ & $63 \pm 2.2^{*}$ & $81 \pm 2.7^{*} \dagger$ & $94 \pm 1.3$ & $73 \pm 3.1^{*}$ & $90 \pm 1.4 \dagger$ & $93 \pm 1.0$ & $73 \pm 2.5^{*}$ & $93 \pm 1.5 \dagger$ \\
\hline
\end{tabular}

$C C P$, Cardioplegic arrest; $C s A$, cyclosporine $A ; H R$, heart rate; $L V D P$, left ventricular developed pressure; $L V E D P$, left ventricular end-diastolic pressure; $d P / d t$, first derivative of left ventricular pressure. Values are mean $\pm \mathrm{SEM} ; \mathrm{n}=6$ each group. ${ }^{*} P<.01$ versus non-CCP. $\dagger P<.01$ versus CCP.

in the current study are consistent with diffusion of cytochrome $\mathrm{c}$ from the mitochondria into the cytosol owing to permeabilization of the outer mitochondrial membrane. Cytochrome c release from mitochondria inhibits mitochondrial respiration owing to limitation of electron transport to cytochrome c oxygenase, ${ }^{26}$ and cytochrome c release, in turn, stimulates mitochondrial superoxide formation that may amplify the deficits in electron transport chain activity. ${ }^{27}$
In addition to these upstream apoptotic signals, Ricci, Gottlieb, and Grier ${ }^{28}$ reported that caspase 3, a "downstream" protease activated by cytochrome c release, can directly inhibit electron transport chain activity. In the present study, the prevention of mitochondrial permeabilization with CsA was associated with preservation of mitochondrial function at complex I, suggesting that loss of reducing equivalents (cytochrome c) or direct inhibition of electron transport can
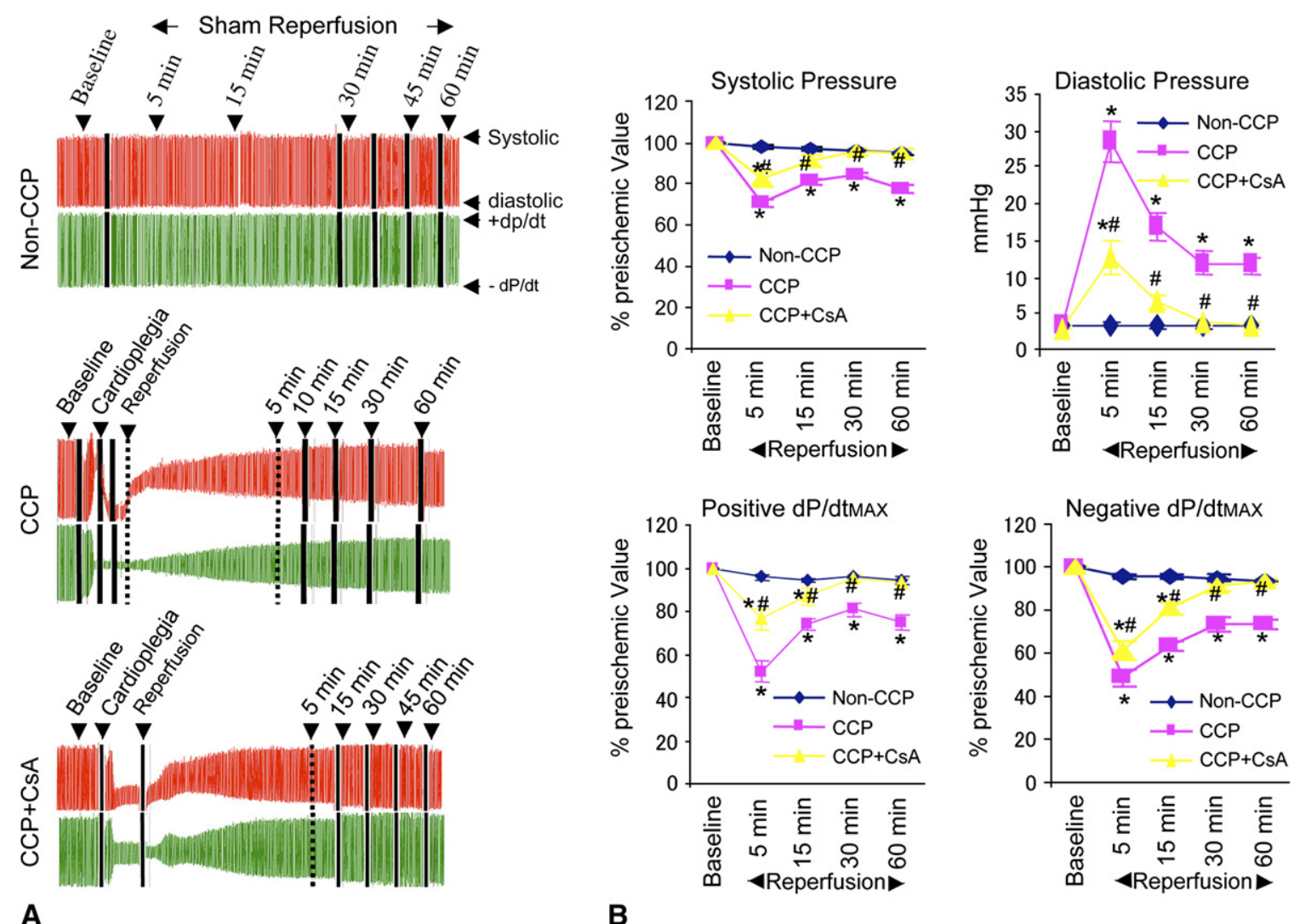

A

Figure 5. A, The representative traces of condensed real-time blood pressure (systolic and diastolic pressure) and first derivatives of left ventricular pressure $( \pm d P / d t)$ from non-CCP, CCP, and CCP $+C s A$ groups. $B$, The changes of systolic pressure, maximum $\pm \mathrm{dP} / \mathrm{dt}$, and the diastolic pressure at 5, 15, 30, and 60 minutes of reperfusion. ${ }^{*} P<.01$ versus non-CCP; \#P<.01 versus CCP. For abbreviations, see Figure 1. 
be prevented with maintenance of mitochondrial outer membrane integrity.

Other investigators have demonstrated that inhibition of mitochondrial dysfunction results in the preservation of myocardial function. ${ }^{29,30}$ Specific inhibition of the MPTP by CsA improves recovery of cardiac function and is associated with reduction of cell death in a variety of in vivo and in vitro ischemia/reperfusion models. ${ }^{17,30}$ Therefore, a rationale is provided for a myopreservation strategy focused on maintenance of mitochondrial structural and functional integrity ${ }^{17,31}$ In the current study, cardioplegic arrest induced conspicuous apoptosis-related alterations in mitochondrial structure and function, and these changes were correlated with myocardial dysfunction. Pretreatment with CsA prevents both ARMD and myocardial dysfunction, supporting a causal relationship between ARMD and global myocardial dysfunction.

Evidence for completion of apoptosis is supported by the increased prevalence of TUNEL-positive nuclei after cardioplegic arrest and abrogation of this finding with cyclosporine. It is important, however, to recognize that positive TUNEL staining is not restricted to apoptotic nuclei and false positive results can occur after stimuli that promote DNA injury (eg, necrosis). $\mathrm{We}^{5,6}$ have previously demonstrated that cardioplegic arrest is associated with DNA laddering, cleavage of caspase 3, and absence of necrosis, which supports the relationship between TUNEL positivity and apoptosis in the current model.

\section{Conclusions}

Inhibition of MPTP opening with CsA prevents ARMD and preserves cardiac global performance after cardioplegic arrest. Better understanding of the interaction between apoptotic signals and ARMD and the correlation of ARMD with myocardial dysfunction will enhance myopreservative strategies for the cardiac surgeon.

\section{References}

1. Crow MT, Mani K, Nam YJ, Kitsis RN. The mitochondrial death pathway and cardiac myocyte apoptosis. Circ Res. 2004;95:957-70.

2. Mootha VK, Wei MC, Buttle KF, Scorrano L, Panoutsakopoulou V, Mannella CA, et al. A reversible component of mitochondrial respiratory dysfunction in apoptosis can be rescued by exogenous cytochrome c. EMBO J. 2001;20:661-71.

3. Appaix F, Guerrero K, Rampal D, Izikki M, Kaambre T, Sikk P, et al. Bax and heart mitochondria: uncoupling and inhibition of respiration without permeability transition. Biochim Biophys Acta. 2002;1556: 155-67.

4. Paradies G, Petrosillo G, Pistolese M, Di Venosa N, Federici A, Ruggerio FM. Decrease in mitochondrial complex I activity in ischemic/reperfused rat heart. Circ Res. 2004;94:53-9.

5. Hammel JM, Caldarone CA, Van Natta TL, Wang L, Welke KF, Li W, et al. Myocardial apoptosis after cardioplegic arrest in the neonatal lamb. J Thorac Cardiovasc Surg. 2003;125:1268-75.

6. Karimi M, Wang L, Hammel JM, Mascio CE, Abdulhamid M, Barner EW, et al. Neonatal vulnerability to ischemia and reperfusion: cardioplegic arrest causes greater myocardial apoptosis in neonatal lambs than in mature lambs. $J$ Thorac Cardiovasc Surg. 2004;127: 490-7.

7. Oka N, Wang L, Mi W, Zhu W, Honjo O, Caldarone CA. Cyclosporine A prevents apoptosis-related mitochondrial dysfunction after cardioplegic arrest. J Thorac Cardiovasc Surg. 2008;135:123-30.

8. Caldarone CA, Barner EW, Wang L, Karimi M, Mascio CE, Hammel JM, et al. Apoptosis-related mitochondrial dysfunction in the early postoperative neonatal lamb heart. Ann Thorac Surg. 2004;78: 948-55.

9. Wang L, Kinnear C, Hammel JM, Zhu W, Hua Z, Mi W, et al. Preservation of mitochondrial structure and function after cardioplegic arrest in the neonate using a selective mitochondrial KATP channel opener. Ann Thorac Surg. 2006;81:1817-23.

10. Garlid KD, Paucek P, Yarov-Yarovoy V, Murray HN, Darbenzio RB, D'Alonzo AJ, et al. Cardioprotective effect of diazoxide and its interaction with mitochondrial ATP-sensitive K+ channels. Possible mechanism of cardioprotection. Circ Res. 1997;81:1072-82.

11. Griffiths EJ, Halestrap AP. Mitochondrial non-specific pores remain closed during cardiac ischaemia, but open upon reperfusion. Biochem J. 1995;307:93-8.

12. Lemasters JJ, Qian T, Elmore SP, Trost LC, Nishimura Y, Herman B, et al. The mitochondrial permeability transition in cell death: a common mechanism in necrosis, apoptosis and autophagy. Biochim Biophys Acta. 1998;1366:177-96.

13. Baines CP, Song CX, Zheng YT, Wang GW, Zhang J, Wang OL, et al. Protein kinase Cepsilon interacts with and inhibits the permeability transition pore in cardiac mitochondria. Circ Res. 2003;92:873-80.

14. Nakagawa $T$, Shimizu $S$, Watanabe $T$, Yamaguchi O, Otsu $K$, Yamagata H, et al. Cyclophilin D-dependent mitochondrial permeability transition regulates some necrotic but not apoptotic cell death Nature. 2005;434:652-8.

15. Akao M, O'Rourke B, Kusuoka H, Teshima Y, Jones SP, Marban E. Differential actions of cardioprotective agents on the mitochondrial death pathway. Circ Res. 2003;92:195-202.

16. Halestrap AP, Connern CP, Griffiths EJ, Kerr PM. Cyclosporin A binding to mitochondrial cyclophilin inhibits the permeability transition pore and protects hearts from ischaemia/reperfusion injury. Mol Cell Biochem. 1997;174:167-72.

17. Argaud L, Gateau-Roesch O, Muntean D, Chalabreysse L, Loufouat J, Robert D, et al. Specific inhibition of the mitochondrial permeability transition prevents lethal reperfusion injury. J Mol Cell Cardiol. 2005; 38:367-74.

18. Nathan M, Friehs I, Choi YH, Cowan DB, Cao-Danh H, McGowan FX, et al. Cyclosporin A but not FK-506 protects against dopamine-induced apoptosis in the stunned heart. Ann Thorac Surg. 2005;79:1620-6.

19. Feng J, Bianchi C, Sandmeyer JL, Li J, Sellke FW. Molecular indices of apoptosis after intermittent blood and crystalloid cardioplegia. Circulation. 2005;112:I184-9.

20. Kowaltowski AJ, Seetharaman S, Paucek P, Garlid KD. Bioenergetic consequences of opening the ATP-sensitive $\mathrm{K}(+)$ channel of heart mitochondria. Am J Physiol Heart Circ Physiol. 2001;280: H649-57.

21. Zinchuk V, Zinchuk O, Okada T. Experimental LPS-induced cholestasis alters subcellular distribution and affects colocalization of Mrp2 and Bsep proteins: a quantitative colocalization study. Microsc Res Tech. 2005;67:65-70.

22. Antonnsson B, Montessuit S, Lauper S, Eskes R, Martinou JC. Bax oligomerization is required for channel-forming activity in liposomes and to trigger cytochrome c release from mitochondria. J Biochem. 2000;345: 271-8.

23. Shimizu S, Narita M, Tsujimoto Y. Bcl-2 family proteins regulate the release of apoptogenic cytochrome $\mathrm{c}$ by the mitochondrial channel VDAC. Nature. 1999;399:483-7.

24. Precht TA, Phelps RA, Linseman DA, Butts BD, Le SS, Laessig TA, et al. The permeability transition pore triggers Bax translocation to mitochondria during neuronal apoptosis. Cell Death Differ. 2005;12: 255-65.

25. Narita M, Shimizu S, Ito T, Chittenden T, Lutz RJ, Matsuda H, et al. Bax interacts with the permeability transition pore to induce permeability 
transition and cytochrome c release in isolated mitochondria. Proc Natl Acad Sci U S A. 1998;95:14681-6.

26. Kuznetzov AV, Schneeberger S, Seiler R, Brandacher G, Mark W, Steurer W, et al. Mitochondrial defects and heterogeneous cytochrome c release after cardiac cold ischemia. Am J Physiol Heart Circ Physiol. 2004;286:H1633-41.

27. Cai J, Yang J, Jones DP. Mitochondrial control of apoptosis: the role of cytochrome c. Biochim Biophys Acta. 1998;1366:139-49.

28. Ricci JE, Gottlieb RA, Green DR. Caspase-mediated loss of mitochondrial function and generation of reactive oxygen species during apoptosis. J Cell Biol. 2003;160:65-75.
29. McCully JD, Wakiyama H, Cowan DB, Federman M, Parker RA, Levitsky S. Diazoxide amelioration of myocardial injury and mitochondrial damage during cardiac surgery. Ann Thorac Surg. 2002;74: 2138-45.

30. Javadov SA, Clarke S, Das M, Griffiths EJ, Lim KH, Halestrap AP. Ischaemic preconditioning inhibits opening of mitochondrial permeability transition pores in the reperfused rat heart. $J$ Physiol. 2003;549:513-24.

31. Rousou AJ, Ericsson M, Federman M, Levitsky S, McCully JD. Opening of mitochondrial KATP channels enhances cardioprotection through the modulation of mitochondrial matrix volume, calcium accumulation, and respiration. Am J Physiol Heart Circ Physiol. 2004;287:H1967-76.

The Journal of Thoracic and Cardiovascular Surgery Conflict of Interest Policy

To assure fairness to authors submitting work for consideration in The Journal of Thoracic and Cardiovascular Surgery, a mechanism exists for managing conflicts of interest. The editor and each of the section editors complete a "Conflict of Interest" form that identifies any and all relationships with commercial and other academic entities. When the editor has a potential conflict because of a relationship with another entity or author, the editor appoints an alternate editor from among the section editors or editorial board members who assumes the entire responsibility for final decisions on the manuscript in question. The editor does not read the reviews that are submitted nor engage in discussing the manuscript prior to the final decision. When the conflict of interest involves a section editor, a "guest section editor" is appointed who fills the role normally played by the conflicted section editor. All members of the editorial board and reviewers are asked to indicate any conflict of interest when they agree to review a manuscript. 